\title{
Lagrange Anchor and Characteristic Symmetries of Free Massless Fields`
}

\author{
Dmitry S. KAPARULIN, Simon L. LYAKHOVICH and Alexey A. SHARAPOV \\ Department of Quantum Field Theory, Tomsk State University, \\ 36 Lenin Ave., Tomsk 634050, Russia \\ E-mail:dsc@phys.tsu.ru, sll@phys.tsu.ru,sharapov@phys.tsu.ru
}

Received December 28, 2011, in final form April 09, 2012; Published online April 12, 2012

http://dx.doi.org/10.3842/SIGMA.2012.021

\begin{abstract}
A Poincaré covariant Lagrange anchor is found for the non-Lagrangian relativistic wave equations of Bargmann and Wigner describing free massless fields of spin $s>1 / 2$ in four-dimensional Minkowski space. By making use of this Lagrange anchor, we assign a symmetry to each conservation law and perform the path-integral quantization of the theory.

Key words: symmetries; conservation laws; Bargmann-Wigner equations; Lagrange anchor

2010 Mathematics Subject Classification: 70S10; 81T70
\end{abstract}

\section{Introduction}

The notions of symmetry and conservation law are of paramount importance for classical and quantum field theory. For Lagrangian theories both the notions are tightly connected to each other due to Noether's first theorem. Beyond the scope of Lagrangian dynamics, this connection has remained unclear, though many particular results and generalizations are known (see [19] for a review). In our recent works $[14,16]$ a general method has been proposed for connecting symmetries and conservation laws in not necessarily Lagrangian field theories. The key ingredient of the method is the notion of a Lagrange anchor introduced earlier [15] in the context of quantization of (non-)Lagrangian dynamics. Geometrically, the Lagrange anchor defines a map from the vector bundle dual to the bundle of equations of motion to the tangent bundle of the configuration space of fields such that certain compatibility conditions are satisfied. In Lagrangian theories, the two bundles coincide and one can take the identity map as a (canonical) Lagrange anchor. For non-Lagrangian field equations, these two bundles may be different and it is a problem by itself to find at least one nontrivial Lagrange anchor, let alone the classification of all admissible Lagrange anchors.

In this paper, the general concept of Lagrange anchor is exemplified by the Bargmann-Wigner equations for free massless fields of $\operatorname{spin} s \geq 1 / 2$ in the four-dimensional Minkowski space [27]. The choice of the example is not accidental. First of all, it has long been known that the model admits infinite sets of rigid symmetries and conservation laws. These have been a subject of intensive studies by many authors during decades, see e.g. [2, 10, 13, 17, 18, 20, 24, 32] and references therein. However, a complete classification has been obtained only recently, first for the conservation laws [3] and then for the symmetries [28]. As the field equations are nonLagrangian for $s>1 / 2$, there is no immediate Noether's correspondence between symmetries and conservation laws. The rich structure of symmetries and conservation laws in the absence

\footnotetext{
* This paper is a contribution to the Special Issue "Symmetries of Differential Equations: Frames, Invariants and Applications". The full collection is available at http://www.emis.de/journals/SIGMA/SDE2012.html
} 
of a Lagrangian formulation makes this theory an ideal testing area for the concept of Lagrange anchor.

The main result of the paper is a Poincaré invariant Lagrange anchor for the BargmannWigner equations. It is well known that each conserved current defines (and is defined by) some characteristic [4, 16, 26]. We have proved earlier [14] that any Lagrange anchor maps characteristics to symmetries. For Lagrangian theories endowed with the canonical Lagrange anchor this map is actually a bijection in accordance with Noether's first theorem. Generally the Lagrange anchor map is neither injective nor surjective. The symmetries that do originate (by the Lagrange anchor map) from characteristics are called characteristic symmetries. In the considered model of the free massless fields, loosely, the characteristic symmetries cover a "half" of equation's symmetries. They also form a subalgebra in the Lie algebra of all the symmetries. To the best of our knowledge the existence of such an infinite dimensional subalgebra in the full Lie algebra of symmetries has not been noticed before for these well studied equations. Furthermore, the pull back of the Lie bracket on characteristic symmetries with respect to the anchor map gives rise to a Lie bracket on the space of conservation laws such that the anchor map appears to be a Lie algebra isomorphism. This bracket generalizes the Dickey bracket [8] of the conserved currents in Lagrangian theory.

For the sake of completeness we also present the quantum probability amplitude on the space of free massless fields which is determined by the proposed Lagrange anchor, and which implements the path-integral quantization of the model.

\section{Equations, symmetries, and characteristics}

\section{$2.1 \quad$ Field equations}

We consider the free massless fields of spin $s \geq 1 / 2$ subject to the relativistic wave-equations

$$
T_{\alpha_{1} \ldots \alpha_{2 s-1}}^{\dot{\alpha}}:=\partial^{\alpha \dot{\alpha}} \varphi_{\alpha \alpha_{1} \ldots \alpha_{2 s-1}}=0,
$$

$\varphi_{\alpha_{1} \ldots \alpha_{2 s}}(x)$ being a symmetric, complex-valued spin-tensor on four-dimensional Minkowski space $\mathbb{R}^{3,1}$. Hereafter we use the standard notation and conventions of the two-component spinor formalism [27]. In particular, $\partial^{\alpha \dot{\alpha}}=\left(\sigma^{\mu}\right)^{\alpha \dot{\alpha}} \partial / \partial x^{\mu}, \mu=0,1,2,3, \alpha, \dot{\alpha}=1,2$, and we raise and lower the spinor indices using the spinor metrics $\varepsilon_{\alpha \beta}, \varepsilon_{\dot{\alpha} \dot{\beta}}$ and their inverse $\varepsilon^{\alpha \beta}, \varepsilon^{\dot{\alpha} \dot{\beta}}$. According to the spin-statistics theorem [29], the fields of integer spin are considered to be bosonic and the fields of half-integer spin are treated as fermionic ${ }^{1}$. Statistics are of no consequence as long as linear in fields expressions are considered (such as equations of motion or symmetry transformations); they, however, become crucial when dealing with nonlinear expressions like quadratic conserved currents.

For $s \geq 1$ the field equations (2.1) satisfy the Noether identities

$$
\partial_{\dot{\alpha}}^{\alpha_{1}} T_{\alpha_{1} \ldots \alpha_{2 s-1}}^{\dot{\alpha}} \equiv 0
$$

though there are no gauge symmetries. This indicates that the equations under consideration are non-Lagrangian save for $s=1 / 2$, as in Lagrangian dynamics there is a one-to-one correspondence between the gauge symmetries and Noether identities (Noether's second theorem [19]).

In what follows we will use some terminology of the geometry of jet spaces, though not systematically. By the $p$ th jet of the field $\varphi$ we mean the following collection of space-time functions:

$$
j^{p} \varphi=\left\{x^{\mu}, \varphi_{\alpha_{1} \ldots \alpha_{2 s}}(x), \partial_{\mu_{1}} \varphi_{\alpha_{1} \ldots \alpha_{2 s}}(x), \ldots, \partial_{\mu_{1}} \cdots \partial_{\mu_{p}} \varphi_{\alpha_{1} \ldots \alpha_{2 s}}(x)\right\} .
$$

\footnotetext{
${ }^{1}$ Notice that in $[3,28]$ all the fields $\varphi_{\alpha_{1} \ldots \alpha_{2 s}}$ are treated as bosonic ones regardless the value of spin.
} 
We say that the space-time function $F$ depends on the $p$ th jet of the field $\varphi$ if it is a smooth function of the elements of $j^{p} \varphi$ considered as independent variables, i.e.,

$$
F=F\left(x^{\mu}, \varphi_{\alpha_{1} \ldots \alpha_{2 s}}(x), \partial_{\mu_{1}} \varphi_{\alpha_{1} \ldots \alpha_{2 s}}(x), \ldots, \partial_{\mu_{1}} \cdots \partial_{\mu_{p}} \varphi_{\alpha_{1} \ldots \alpha_{2 s}}(x)\right) .
$$

We will also refer to $F$ as a local function of fields.

\subsection{Symmetries}

A variational vector field

$$
Z=\int d^{4} x\left(Z_{\alpha_{1} \ldots \alpha_{2 s}} \frac{\delta}{\delta \varphi_{\alpha_{1} \ldots \alpha_{2 s}}(x)}+\text { c.c. }\right)
$$

is called a symmetry of order $p$ if its components $Z_{\alpha_{1} \ldots \alpha_{2 s}}$ depend on the $p$ th jet of the field $\varphi$ and the following condition is satisfied:

$$
\left.Z T_{\alpha_{1} \ldots \alpha_{2 s-1}}^{\dot{\alpha}}\right|_{T_{s}=0}=0
$$

where $T_{s}=0$ is a shorthand notation for (2.1). The last relation means that the variation of the field equations (2.1) along $Z$ is given by a linear combination of these equations and their differential consequences. In other words, the local function of fields $Z T_{s}$ vanishes on every solution to the field equations. A symmetry is called trivial if

$$
\left.Z_{\alpha_{1} \ldots \alpha_{2 s}}\right|_{T_{s}=0}=0
$$

All the symmetries below are considered modulo trivial ones.

A symmetry $Z$ is called elementary if the functions $Z_{\alpha_{1} \ldots \alpha_{2 s}}$ are independent of fields, i.e., they only depend on $x$ 's. Elementary symmetries correspond to shifts of the field $\varphi$ by a particular solution of the equations of motion. Clearly, such symmetries have a place in any linear theory.

In [28], it was shown that all non-elementary symmetries of equations (2.1) are generated by variational vector fields (2.3) whose coefficients $Z_{\alpha_{1} \ldots \alpha_{2 s}}$ can be chosen to be linear in fields and their derivatives. For this reason we refer to these symmetries as linear and denote the space of all linear symmetries by $\operatorname{Sym}\left(T_{s}\right)$. There is an increasing filtration of $\operatorname{Sym}\left(T_{s}\right)$ by the subspaces of linear symmetries of order $p$, namely,

$$
0=\operatorname{Sym}_{-1}\left(T_{s}\right) \subset \operatorname{Sym}_{0}\left(T_{s}\right) \subset \operatorname{Sym}_{1}\left(T_{s}\right) \subset \cdots \subset \operatorname{Sym}_{\infty}\left(T_{s}\right)=\operatorname{Sym}\left(T_{s}\right) .
$$

It turns out that $\operatorname{dim} \operatorname{Sym}_{p}\left(T_{s}\right)<\infty$ for all $p=0,1, \ldots$ The generators of symmetry transformations $Z$ define (and are defined by) some linear endomorphisms $\hat{Z}$ of the space of fields. Linearity of the field equations (2.1) and the condition (2.4) suggest that $\hat{Z}$ are precisely those endomorphisms that are interchangeable with the wave operator $\hat{T}_{s}$ of equations (2.1) in the following sense:

$$
\hat{T}_{s} \hat{Z}=\hat{A} \hat{T}_{s}
$$

for some matrix differential operator $\hat{A}$. As a result, the space $\operatorname{Sym}\left(T_{s}\right)$ carries the structure of an associative filtered algebra ${ }^{2}$ with respect to the composition of endomorphisms underlying the symmetries. The corresponding product

$$
\text { * } \operatorname{Sym}_{p}\left(T_{s}\right) \times \operatorname{Sym}_{q}\left(T_{s}\right) \rightarrow \operatorname{Sym}_{p+q}\left(T_{s}\right)
$$

\footnotetext{
${ }^{2}$ For a general theory of filtered and graded algebras we refer the reader to [25].
} 
can be written as follows: If $Z^{\prime}$ and $Z^{\prime \prime}$ are two linear symmetries, then $Z^{\prime} * Z^{\prime \prime}$ is a linear symmetry generated by the variational vector field (2.3) with components

$$
Z_{\alpha_{1} \ldots \alpha_{2 s}}=Z^{\prime}\left(Z_{\alpha_{1} \ldots \alpha_{2 s}}^{\prime \prime}\right) \text {. }
$$

Associated to the filtered algebra (2.5) is the graded algebra

$$
\operatorname{GrSym}\left(T_{s}\right)=\bigoplus_{p=0}^{\infty} \operatorname{Sym}^{p}\left(T_{s}\right), \quad \operatorname{Sym}^{p}\left(T_{s}\right)=\operatorname{Sym}_{p}\left(T_{s}\right) / \operatorname{Sym}_{p-1}\left(T_{s}\right) .
$$

Although the algebras $\operatorname{Sym}\left(T_{s}\right)$ and $\operatorname{GrSym}\left(T_{s}\right)$ are not isomorphic to each other, there is an isomorphism of filtered vector spaces

$$
\operatorname{GrSym}\left(T_{s}\right) \simeq \operatorname{Sym}\left(T_{s}\right)
$$

where the filtration in $\operatorname{GrSym}\left(T_{s}\right)$ is given by the standard filtration of a graded vector space:

$$
\operatorname{GrSym}_{p}\left(T_{s}\right) \subset \operatorname{GrSym}_{p+1}\left(T_{s}\right), \quad \operatorname{GrSym}_{p}\left(T_{s}\right)=\bigoplus_{k=0}^{p} \operatorname{Sym}^{k}\left(T_{s}\right), \quad p=0,1, \ldots
$$

The isomorphism (2.6) is far from being canonical, but it implies the equality

$$
\operatorname{dim} \operatorname{GrSym}_{p}\left(T_{s}\right)=\operatorname{dim} \operatorname{Sym}_{p}\left(T_{s}\right) .
$$

As for any associative algebra, we can turn $\operatorname{Sym}\left(T_{s}\right)$ into a filtered Lie algebra with respect to the $*$-commutator

$$
\left[Z_{1}, Z_{2}\right]=Z_{1} * Z_{2}-Z_{2} * Z_{1}
$$

which is actually given by the commutator of variational vector fields. Whereas the closedness with respect to commutation is an inherent property of the infinitesimal symmetry transformations, the existence of an associative $*$-product on symmetries is a special property of linear field equations.

\subsection{Conserved currents and characteristics}

A conserved current of order $p$ is a real vector-function $\Phi^{\alpha \dot{\alpha}}$ that depends on the $p$ th jet of the field $\varphi$ and satisfies the condition

$$
\left.\partial_{\alpha \dot{\alpha}} \Phi^{\alpha \dot{\alpha}}\right|_{T_{s}=0}=0
$$

A conserved current $\Phi$ is called trivial if, being evaluated on solutions to the field equations, it is given by the divergence of some bivector field, that is,

$$
\Phi^{\alpha \dot{\alpha}}=\partial_{\beta}^{\dot{\alpha}} \Theta^{\alpha \beta}+\text { c.c. } \quad\left(\bmod T_{s}\right)
$$

for some local functions of fields $\Theta^{\alpha \beta}=\Theta^{\beta \alpha}$. The trivial currents, having zero charge, are of no physical importance. Therefore, we consider the conserved currents modulo trivial ones. It is easy to see that in each equivalence class of a conserved current there is a representative obeying the relation

$$
\partial_{\alpha \dot{\alpha}} \Phi^{\alpha \dot{\alpha}}=T_{\alpha_{1} \ldots \alpha_{2 s-1}}^{\dot{\alpha}} Q_{\dot{\alpha}}^{\alpha_{1} \ldots \alpha_{2 s-1}}+\text { c.c. }
$$


where $Q_{\dot{\alpha}}^{\alpha_{1} \ldots \alpha_{2 s-1}}$ is a local function of fields. The spinor-valued function $Q$ is called the characteristic of the conserved current $\Phi$. We say that a characteristic is of order $p$ if it depends on the $p$ th jet of the field $\varphi$.

The existence of the Noether identities (2.2) gives rise to a great number of characteristics of the form

$$
Q_{\dot{\alpha}}^{\alpha_{1} \ldots \alpha_{2 s-1}}=\partial_{\dot{\alpha}}^{\left(\alpha_{1}\right.} \chi^{\left.\alpha_{2} \ldots \alpha_{2 s-1}\right)},
$$

where $\chi$ is an arbitrary symmetric spin-tensor field. All these characteristics correspond to trivial conserved currents (2.7) and can be ignored. Another source of triviality is characteristics that are proportional to the (differential consequence of) field equations. Such characteristics also result in trivial conserved currents and should be regarded as trivial. Taking the quotient of the space of all characteristics by the subspace of trivial ones, we get the space of nontrivial characteristics.

Actually, relation (2.8) gives rise to a one-to-one correspondence between the spaces of nontrivial conserved currents and characteristics, when either is properly defined. For the nondegenerate systems of PDEs such an isomorphism has been known for a long time, see e.g. [26]. Its extension to the Lagrangian gauge systems was given in [4], using some previous results on the local BRST cohomology [9]. A further generalization to the case of arbitrary (i.e., not necessarily Lagrangian and/or non-degenerate) system of PDEs subject to the standard regularity conditions [4] was formulated in our recent paper [16]. Thus, for regular PDEs the study of conservation laws amounts to the study of characteristics and vice versa. For the Bargmann-Wigner equations (2.1) this general isomorphism was traced explicitly in [3].

In [28], it was shown that all the nontrivial characteristics for equations (2.1) can be chosen to be at most linear in fields. Correspondingly, all the nontrivial conserved currents can be chosen to be at most quadratic in fields. Below we are restricted to the characteristics with linear dependence of fields. These form the linear space Char $\left(T_{s}\right)$ filtered by the increasing sequence of finite dimensional subspaces $\operatorname{Char}_{p}\left(T_{s}\right)$ constituted by the characteristics of order $p$,

$$
0=\operatorname{Char}_{-1}\left(T_{s}\right) \subset \operatorname{Char}_{0}\left(T_{s}\right) \subset \operatorname{Char}_{1}\left(T_{s}\right) \subset \cdots \subset \operatorname{Char}_{\infty}\left(T_{s}\right)=\operatorname{Char}\left(T_{s}\right) .
$$

Taking the successive quotients

$$
\operatorname{Char}^{p}\left(T_{s}\right)=\operatorname{Char}_{p}\left(T_{s}\right) / \operatorname{Char}_{p-1}\left(T_{s}\right)
$$

we define the associated graded space of linear characteristics

$$
\operatorname{GrChar}\left(T_{s}\right)=\bigoplus_{p=0}^{\infty} \operatorname{Char}^{p}\left(T_{s}\right)
$$

As with the symmetries, there is an isomorphism of filtered vector spaces

$$
\operatorname{GrChar}\left(T_{s}\right) \simeq \operatorname{Char}\left(T_{s}\right)
$$

with respect to the canonical filtration in $\operatorname{GrChar}\left(T_{s}\right)$,

$$
\operatorname{GrChar}_{p}\left(T_{s}\right) \subset \operatorname{GrChar}_{p+1}\left(T_{s}\right), \quad \operatorname{GrChar}_{p}\left(T_{s}\right)=\bigoplus_{k=0}^{p} \operatorname{Char}^{p}\left(T_{s}\right) .
$$

In particular,

$$
\operatorname{dim} \operatorname{GrChar}_{p}\left(T_{s}\right)=\operatorname{dim} \operatorname{Char}_{p}\left(T_{s}\right) .
$$


Characteristics form a filtered module over the filtered Lie algebra $\operatorname{Sym}\left(T_{s}\right)$. The action of symmetries on characteristics

$$
\odot: \operatorname{Sym}_{p}\left(T_{s}\right) \times \operatorname{Char}_{q}\left(T_{s}\right) \rightarrow \operatorname{Char}_{p+q}\left(T_{s}\right)
$$

can be defined as follows. Let $Z$ be a symmetry and let $\Phi$ be the conserved current associated to a characteristic $Q$. Then $\Phi^{\prime}=Z \Phi$ is a new conserved current and we define $Q^{\prime}=Z \odot Q$ to be the characteristic corresponding to $\Phi^{\prime}$. Due to the one-to-one correspondence between the spaces of nontrivial currents and nontrivial characteristics the above definition is consistent. It should be noted that the space $\operatorname{Char}\left(T_{s}\right)$, being a module over the Lie algebra $\operatorname{Sym}\left(T_{s}\right)$, is by no means a module over the associative algebra $\left(\operatorname{Sym}\left(T_{s}\right), *\right)$.

\section{Classification results}

As was mentioned in the Introduction a complete classification of the rigid symmetries and conservation laws for purely bosonic fields (2.1) of spin $s \geq 1 / 2$ was given by Anco and Pohjanpelto in [3, 28] (see also [32]). Below we give a brief summary of these results and comment on a difference between the bosonic and fermionic cases. An important part of the classification is played by the algebra of Killing spinors on the four-dimensional Minkowski space. Therefore we start with recalling some basic facts concerning this algebra.

\subsection{The Killing spinors}

By definition, a Killing spinor is a spin-tensor field $\xi_{\dot{\alpha}_{1} \ldots \dot{\alpha}_{l}}^{\alpha_{1} \ldots \alpha_{k}}(x)$ of type $(k, l)$ which is symmetric in dotted and undotted indices and obeys the equation

$$
\partial_{(\dot{\alpha}}^{(\alpha} \xi_{\left.\dot{\alpha}_{1} \ldots \dot{\alpha}_{l}\right)}^{\left.\alpha_{1} \ldots \alpha_{k}\right)}=0
$$

As above, the round brackets mean symmetrization of the enclosed indices.

Let $\operatorname{Kil}(k, l)$ denote the space of all Killing spinors of type $(k, l)$. The Killing spinors form a bigraded, associative, commutative algebra $\mathrm{Kil}=\bigoplus_{k, l \in \mathbb{N}} \mathrm{Kil}(k, l)$ with respect to the symmetrized tensor product

$$
\text { - : } \operatorname{Kil}(k, l) \times \operatorname{Kil}\left(k^{\prime}, l^{\prime}\right) \rightarrow \operatorname{Kil}\left(k+k^{\prime}, l+l^{\prime}\right) .
$$

If $\xi \in \operatorname{Kil}(k, l)$ and $\xi^{\prime} \in \operatorname{Kil}\left(k^{\prime}, l^{\prime}\right)$ then $\xi \circ \xi^{\prime}$ is the Killing spinor of type $\left(k+k^{\prime}, l+l^{\prime}\right)$ with components

$$
\left(\xi \bullet \xi^{\prime}\right)_{\dot{\alpha}_{1} \ldots \dot{\alpha}_{l+l^{\prime}}}^{\alpha_{1} \ldots \alpha_{k+k^{\prime}}}=\xi_{\left(\dot{\alpha}_{1} \ldots \dot{\alpha}_{l}\right.}^{\left(\alpha_{1} \ldots \alpha_{k}\right.} \xi_{\left.\dot{\alpha}_{l+1} \ldots \dot{\alpha}_{l+l^{\prime}}\right)}^{\left.\alpha_{k+1} \ldots \alpha_{k+k^{\prime}}\right)}
$$

The complex conjugation of spin-tensor fields, $\xi \mapsto \bar{\xi}$, defines an involution in the algebra Kil such that $\overline{\operatorname{Kil}(k, l)} \simeq \operatorname{Kil}(l, k)$. The Killing spinor $\xi$ of type $(s, s)$ is called real if $\bar{\xi}=\xi$. The space of all real spinors of type $(s, s)$ will be denoted by $\operatorname{Kil}_{\mathbb{R}}(s, s)$.

In what follows we will be mostly interested in Killing spinors belonging to the following sequence of vector spaces

$$
\mathrm{Kil}_{s}=\bigoplus_{p=0}^{\infty} \mathrm{Kil}_{s}^{p}, \quad \mathrm{Kil}_{s}^{p}=\operatorname{Kil}(p, p) \oplus \operatorname{Kil}(p-2 s, p+2 s), \quad s=\frac{1}{2}, 1, \frac{3}{2}, 2, \ldots
$$

Here it is implied that $\operatorname{Kil}(p-2 s, p+2 s)=0$ whenever $2 s>p$. It is well known that each homogeneous subspace of $\mathrm{Kil}_{s}$ is of finite dimension, namely,

$$
\operatorname{dim}_{\mathbb{C}} \operatorname{Kil}(p-2 s, p+2 s)=(p-2 s+1)(p-2 s+2)(p+2 s+1)(p+2 s+2)(2 p+3) / 12 .
$$


Although the space $\mathrm{Kil}_{s}$ is not a subalgebra with respect to the --product (3.1), it can be endowed with the structure of an associative superalgebra with respect to a new product

$$
\circ: \mathrm{Kil}_{s}^{p} \times \mathrm{Kil}_{s}^{q} \rightarrow \mathrm{Kil}_{s}^{p+q} .
$$

The $\mathbb{Z}_{2}$-grading on $\mathrm{Kil}_{s}$ is defined by the direct sum decomposition

$$
\mathrm{Kil}_{s}=\mathrm{Kil}_{s}^{+} \oplus \mathrm{Kil}_{s}^{-},
$$

where

$$
\mathrm{Kil}_{s}^{+}=\bigoplus_{p=0}^{\infty} \operatorname{Kil}(p, p), \quad \mathrm{Kil}_{s}^{-}=\bigoplus_{p=0}^{\infty} \operatorname{Kil}(p, p+4 s) .
$$

Then for all $\xi, \xi^{\prime} \in \mathrm{Kil}_{s}^{+}$and $\eta, \eta^{\prime} \in \mathrm{Kil}_{s}^{-}$we set

$$
\xi \circ \xi^{\prime}=\xi \bullet \xi^{\prime}, \quad \xi \circ \eta=\bar{\xi} \bullet \eta, \quad \eta \circ \xi=\eta \bullet \xi, \quad \eta \circ \eta^{\prime}=\bar{\eta} \bullet \eta^{\prime} .
$$

One can easily verify that, being defined in such a way, the o-product is associative, though not commutative. At the same time, its restriction to the even subalgebra $\mathrm{Kil}_{s}^{+}$coincides with the -product and is thus commutative. Involving the complex conjugation, the o-product is only $\mathbb{R}$-linear, not $\mathbb{C}$-linear.

As an associative algebra the subalgebra $\mathrm{Kil}_{s}^{+}$is generated by the Killing spinors of type $(1,1)$, so that each even element can be represented as a sum of elements of the form

$$
\xi_{1} \circ \xi_{2} \circ \cdots \circ \xi_{p}, \quad \xi_{i} \in \operatorname{Kil}(1,1) .
$$

Similarly, each element from the odd subspace $\mathrm{Kil}_{s}^{-}$is given by a linear combination of the Killing spinors

$$
\xi_{1} \circ \xi_{2} \circ \cdots \circ \xi_{p} \circ \Upsilon, \quad \Upsilon \in \operatorname{Kil}(0,4 s) .
$$

Having in mind the standard relation between tensor and spin-tensor fields in four-dimensional Minkowski space, we refer to type $(p, p)$ Killing spinors as Killing tensors of rank $p$ and to type $(0,4 s)$ Killing spinor as self-dual Killing-Yano tensors of rank $2 s$. The Killing tensors of rank 1 are just the Killing vectors.

\subsection{Classification}

A general correspondence between the space of Killing spinors, nontrivial symmetries and characteristics can be summarized by the diagram of maps

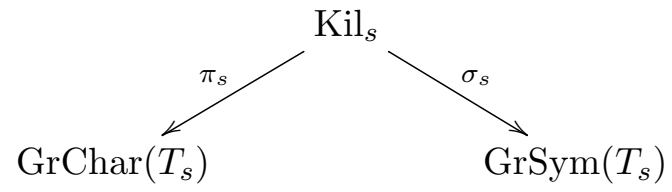

with the following properties:

1. The homomorphisms $\pi_{s}$ and $\sigma_{s}$ are homogeneous of degrees $1-2 s$ and 0 , respectively. Hence,

$$
\pi_{s}: \operatorname{Kil}_{s}^{p} \rightarrow \operatorname{Char}^{p-2 s+1}\left(T_{s}\right), \quad \sigma_{s}: \operatorname{Kil}_{s}^{p} \rightarrow \operatorname{Sym}^{p}\left(T_{s}\right) .
$$


2. $\sigma_{s}$ is an isomorphism of graded associative algebras.

3. $\pi_{s}$ is a surjection.

Let us first describe the homomorphism $\sigma_{s}$. By definition, it takes the generators

$$
1 \in \operatorname{Kil}(0,0), \quad \xi \in \operatorname{Kil}(1,1), \quad \Upsilon \in \operatorname{Kil}(0,4 s)
$$

of the algebra $\mathrm{Kil}_{s}$ to the variational vector field (2.3) with components

$$
\begin{aligned}
& Z[1]_{\alpha_{1} \ldots \alpha_{2 s}}=\varphi_{\alpha_{1} \ldots \alpha_{2 s}}, \\
& Z[\xi]_{\alpha_{1} \ldots \alpha_{2 s}}=\xi^{\beta \dot{\beta}} \partial_{\beta \dot{\beta}} \varphi_{\alpha_{1} \ldots \alpha_{2 s}}+s \partial_{\left(\alpha_{1} \dot{\beta}\right.} \xi^{\beta \dot{\beta}} \varphi_{\left.\beta \alpha_{2} \ldots \alpha_{2 s}\right)}+\frac{1-s}{4}\left(\partial_{\beta \dot{\beta}} \xi^{\beta \dot{\beta}}\right) \varphi_{\alpha_{1} \ldots \alpha_{2 s}}, \\
& Z[\Upsilon]_{\alpha_{1} \ldots \alpha_{2 s}}=\sum_{p=0}^{2 s} \frac{2 s-p+1}{4 s+1}\left(\begin{array}{c}
2 s \\
p
\end{array}\right)\left(\partial_{\left(\alpha_{1}\right.}^{\dot{\beta}_{1}} \ldots \partial_{\alpha_{p}}^{\dot{\beta}_{p}} \Upsilon_{\dot{\beta}_{1} \ldots \dot{\beta}_{4 s}}\right)\left(\partial_{\alpha_{p+1}}^{\dot{\beta}_{p+1}} \cdots \partial_{\left.\alpha_{2 s}\right)}^{\dot{\beta}_{2 s}} \bar{\varphi}^{\dot{\beta}_{2 s+1} \ldots \dot{\beta}_{4 s}}\right) .
\end{aligned}
$$

Clearly, $Z[1]$ generates the dilatations of the field $\varphi$ and $Z[\xi]$ corresponds to the "conformally weighted" Lie derivative of $\varphi$ along the Killing vector $\xi$. By property (2) the homomorphism $\sigma_{s}$ is uniquely extended from the multiplicative (3.4) to linear generators (3.2), (3.3) of the algebra $\mathrm{Kil}_{s}$ :

$$
\begin{aligned}
& Z\left[\xi_{1} \circ \cdots \circ \xi_{p}\right]=Z\left[\xi_{1}\right] * \cdots * Z\left[\xi_{p}\right], \\
& Z\left[\zeta_{1} \circ \cdots \circ \zeta_{p-2 s} \circ \Upsilon\right]=Z\left[\zeta_{1}\right] * \cdots * Z\left[\zeta_{p-2 s}\right] * Z[\Upsilon]
\end{aligned}
$$

Hereafter we use the Greek letter $\zeta$ to denote the real Killing vectors, i.e., elements of $\mathrm{Kil}_{\mathbb{R}}(1,1)$, while $\xi$ is used for complex Killing vectors. Notice that the symmetries in the right hand sides of (3.5) represent elements of the graded vector space $\operatorname{GrSym}\left(T_{s}\right)$ and, as elements of $\operatorname{GrSym}\left(T_{s}\right)$, are completely symmetric in permutations of $\xi$ 's and $\zeta$ 's. The last fact easily follows from the commutation relations

$$
\left[Z\left[\xi_{1}\right], Z\left[\xi_{2}\right]\right]=Z\left[\left[\xi_{1}, \xi_{2}\right]\right], \quad[Z[\Upsilon], Z[\zeta]]=Z\left[\mathcal{L}_{\zeta} \Upsilon\right]
$$

The symmetries (3.5) exhaust all the non-elementary symmetries of the relativistic wave equation (2.1). The space of symmetries inherits the $\mathbb{Z}_{2}$-grading from the space of Killing tensors, namely, the even symmetries take the field to field, while the odd symmetries take the field to its complex conjugate.

Let us now turn to the homomorphism $\pi_{s}$. Again, the $\mathbb{Z}_{2}$-grading on the space $\mathrm{Kil}_{s}$ induces the same grading on the space of characteristics. As a $\mathbb{Z}_{2}$-graded module over the Lie algebra of symmetries the space $\operatorname{GrChar}\left(T_{s}\right)$ is generated by the even characteristics of order zero

$$
Q\left[i^{2 s} \zeta_{1} \circ \cdots \circ \zeta_{2 s-1}\right]_{\alpha}^{\dot{\alpha}_{1} \ldots \dot{\alpha}_{2 s-1}}=\left(\zeta_{1} \circ \cdots \circ \zeta_{2 s-1}\right)^{\alpha_{1} \ldots \alpha_{2 s-1} \dot{\alpha}_{1} \ldots \dot{\alpha}_{2 s-1}} \varphi_{\alpha \alpha_{1} \ldots \alpha_{2 s-1}}
$$

and some odd characteristics of order one or two depending on $s$. For half-integer spins the latter characteristics are given by

$$
\begin{aligned}
Q\left[i^{2 s} \Upsilon\right]_{\alpha}^{\dot{\alpha}_{1} \ldots \dot{\alpha}_{2 s-1}}= & \Upsilon^{\dot{\alpha} \dot{\alpha}_{1} \ldots \dot{\alpha}_{2 s-1} \dot{\beta}_{1} \ldots \dot{\beta}_{2 s}} \partial_{\alpha \dot{\alpha}} \bar{\varphi}_{\dot{\beta}_{1} \ldots \dot{\beta}_{2 s}} \\
& +\frac{2 s+1}{4 s+1} \partial_{\alpha \dot{\alpha}} \Upsilon^{\dot{\alpha} \dot{\alpha}_{1} \ldots \dot{\alpha}_{2 s-1} \dot{\beta}_{1} \ldots \dot{\beta}_{2 s}} \bar{\varphi}_{\dot{\beta}_{1} \ldots \dot{\beta}_{2 s}}
\end{aligned}
$$

and for integer spins they can be written as

$$
Q[\zeta \circ \Upsilon]=Z[\zeta] Q[\Upsilon]+\frac{1}{2} Q\left[\mathcal{L}_{\zeta} \Upsilon\right]
$$


Now the space of characteristics for real-valued conserved currents is spanned by the even characteristics

$$
Q\left[i^{q+2 s} \zeta_{1} \circ \cdots \circ \zeta_{q+2 s-1}\right]=Z\left[i^{q} \zeta_{1} \circ \cdots \circ \zeta_{q}\right] \odot Q\left[i^{2 s} \zeta_{q+1} \circ \cdots \circ \zeta_{q+2 s-1}\right]
$$

and odd characteristics

$$
\begin{array}{ll}
Q\left[\zeta_{1} \circ \cdots \circ \zeta_{2 p} \circ \Upsilon\right]=Z\left[\zeta_{1} \circ \cdots \circ \zeta_{2 p}\right] \odot Q[\Upsilon], & s \in \mathbb{N}-\frac{1}{2}, \\
Q\left[\zeta_{1} \circ \cdots \circ \zeta_{2 p+1} \circ \Upsilon\right]=Z\left[\zeta_{1} \circ \cdots \circ \zeta_{2 p}\right] \odot Q\left[\zeta_{2 p+1} \circ \Upsilon\right], & s \in \mathbb{N} .
\end{array}
$$

The characteristics (3.9), (3.10) have the orders $q, 2 p+1$ and $2 p+2$, respectively. Let us stress again that the expressions in the right hand sides of (3.9) and (3.10) are considered as representing elements of $\operatorname{GrChar}\left(T_{s}\right)$, i.e., modulo characteristics of lower orders. Upon this interpretation the right hand sides are totally symmetric in $\zeta$ 's.

Formulae (3.6)-(3.10) provide a complete description of the homomorphism $\pi_{s}$. In particular, they show that $\pi_{s}$ is surjective. The kernel of $\pi_{s}$ is given by the space

$$
\operatorname{Ker} \pi_{s}=\mathrm{Ker}_{+} \pi_{s} \oplus \mathrm{Ker}_{-} \pi_{s}
$$

where

$$
\operatorname{Ker}_{+} \pi_{s}=\left(\bigoplus_{n=0}^{2 s-2} \operatorname{Kil}(n, n)\right) \oplus\left(\bigoplus_{m=2 s-1}^{\infty} i^{m} \operatorname{Kil}_{\mathbb{R}}(m, m)\right)
$$

and

$$
\operatorname{Ker}_{-} \pi_{s}= \begin{cases}\bigoplus_{p=0}^{\infty} \operatorname{Kil}(2 p+1,2 p+4 s+1), & \text { for } s \in \mathbb{N}-\frac{1}{2} \\ \bigoplus_{p=0}^{\infty} \operatorname{Kil}(2 p, 2 p+4 s), & \text { for } s \in \mathbb{N} .\end{cases}
$$

Introduce the subspace $\mathrm{ChKil}_{s} \subset \mathrm{Kil}_{s}$ which is complementary to $\operatorname{Ker} \pi_{s}$ :

$$
\mathrm{ChKil}_{s}=\mathrm{ChKil}_{s}^{+} \oplus \mathrm{ChKil}_{s}^{-},
$$

where

$$
\mathrm{ChKil}_{s}^{+}=\bigoplus_{m=2 s-1}^{\infty} i^{m+1} \operatorname{Kil}_{\mathbb{R}}(m, m)
$$

and

$$
\mathrm{ChKil}_{s}^{-}= \begin{cases}\bigoplus_{p=0}^{\infty} \operatorname{Kil}(2 p, 2 p+4 s), & \text { for } s \in \mathbb{N}-\frac{1}{2} \\ \bigoplus_{p=0}^{\infty} \operatorname{Kil}(2 p+1,2 p+4 s+1), & \text { for } s \in \mathbb{N}\end{cases}
$$

With the definitions above we have the direct sum decomposition

$$
\mathrm{Kil}_{s}=\operatorname{Ker} \pi_{s} \oplus \mathrm{ChKil}_{s}
$$


and $\pi_{s}$ becomes an isomorphism when restricted to $\mathrm{ChKil}_{s}$. So, we can draw the following diagram:

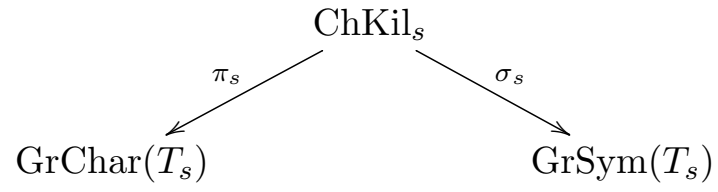

where $\sigma_{s}$ is sill an injection. For an obvious reason we refer to $\mathrm{ChKil}_{s}$ as the space of characteristic Killing tensors. As is seen, no characteristics are assigned to the Killing tensors of rank $<2 s-1$ and only the odd (even) rank tensors of Killing and Yano correspond to characteristics for integer (half-integer) spins.

Remark 1. The original classification [3] of the quadratic conserved currents was formulated for purely bosonic fields. It essentially relied on the notion of an adjoint symmetry, see equation (A.2) of Appendix A. Each Killing spinor $\xi \in \mathrm{Kil}_{s}^{p}, p \geq 2 s-1$, was shown to give rise to an adjoint symmetry and a conserved current. By construction, such currents exhaust all the quadratic conserved currents, but dependencies are allowed (i.e., some of nontrivial linear combinations of the conserved currents may give a trivial current). Actually, only a "half" of the Killing spinors above was shown to generate a basis of the nontrivial conserved currents [3, Corollary 4.4]. This classification method is directly applicable to the fermionic fields as well, but the resulting conserved currents are different. For the fermionic fields of half-integer spin, exactly the complementary "half" of the Killing spinors spans the space of nontrivial quadratic currents. Expressions (3.9), (3.10) are given for the standard spin-statistics correspondence. Another approach to the construction of conserved currents for massless fields has been proposed in [32]. In that paper, the conserved currents have been defined as contractions of conserved tensors with appropriate Killing spinors, so that the map $\pi_{s}$ has appeared in quite a natural way. However, the dependencies have been unnoticed between the currents, and the kernel of $\pi_{s}$ was not studied. With a due account of these dependencies and the statistics, the real-valued conserved currents found in [32] coincide with those constructed and classified in [3].

\section{Lagrange anchor and characteristic symmetries}

The Lagrange anchor was first introduced in [15] as a tool for the path-integral quantization of non-Lagrangian theories. Later, it has been realized [14] that the concept of Lagrange anchor can also serve in classical theory for establishing a systematic correspondence between conservation laws and symmetries, providing a generalization of the first Noether's theorem to non-Lagrangian dynamics. The latter result admits also a natural cohomological interpretation within the BRST formalism [16]. In the body of the paper, we do not elaborate on the definition and general properties of the Lagrange anchor, which are explained from various viewpoints in the cited works. A brief account of the general notions concerning the Lagrange anchor can be found in Appendix A.

In this particular model, one can arrive at the Lagrange anchor proceeding from the following simple observation. Since the map $\pi_{s}: \mathrm{ChKil}_{s} \rightarrow \operatorname{GrChar}\left(T_{s}\right)$ is an isomorphism, the diagram (3.11) can be completed uniquely to the commutative triangle diagram

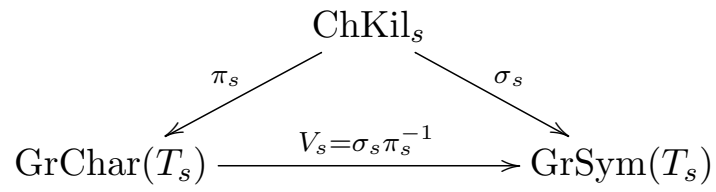


Now, one can see that the bottom map is given by a universal linear differential operator acting from the space of characteristics to the space of symmetries. It is the operator that can be taken as a Lagrange anchor for the field equations (2.1). Explicitly, if $Q=\left(Q_{\dot{\alpha}}^{\alpha_{1} \ldots \alpha_{2 s-1}}\right)$ is a characteristic, then $V_{s}$ takes it to the symmetry (2.3) with

$$
Z_{\alpha_{1} \ldots \alpha_{2 s}}=V_{s}(Q)_{\alpha_{1} \ldots \alpha_{2 s}}=i^{2 s} \partial_{\left(\alpha_{2} \dot{\alpha}_{2}\right.} \ldots \partial_{\alpha_{2 s-1} \dot{\alpha}_{2 s-1}} \bar{Q}_{\left.\alpha_{1}\right)}^{\dot{\alpha}_{2} \ldots \dot{\alpha}_{2 s}} .
$$

Verification of the defining property of a Lagrange anchor (A.5) is straightforward, see Appendix B. Actually, formula (4.2), considered for particular representatives of characteristics and symmetries, defines the map

$$
V_{s}: \operatorname{Char}_{p}\left(T_{s}\right) \rightarrow \operatorname{Sym}_{p+2 s-1}\left(T_{s}\right), \quad p=0,1,2, \ldots,
$$

of the underlying filtered spaces, from which the induced map (4.1) of the associated graded spaces follows. (By abuse of notation, we denote both the maps by $V_{s}$.) The map (4.3), being defined through the composition of monomorphisms $\sigma_{s}$ and $\pi_{s}^{-1}$, is obviously injective and we denote its image by $\operatorname{ChSym}_{p}\left(T_{s}\right)$. The space $\operatorname{ChSym}\left(T_{s}\right)=\operatorname{ChSym}_{\infty}\left(T_{s}\right)$ is referred to as the space of characteristic symmetries.

Being independent of fields, the Lagrange anchor $V_{s}$ is automatically strongly integrable, see relations (A.7) of Appendix A. The last fact implies that the characteristic symmetries form a subalgebra in the Lie algebra $\operatorname{Sym}\left(T_{s}\right)$. We have

$$
\left[V_{s}\left(Q_{1}\right), V_{s}\left(Q_{2}\right)\right]=V_{s}\left(\left[Q_{1}, Q_{2}\right]_{V_{s}}\right), \quad \forall Q_{1}, Q_{2} \in \operatorname{Char}\left(T_{s}\right),
$$

where

$$
\left[Q_{1}, Q_{2}\right]_{V_{s}}:=V_{s}\left(Q_{1}\right) \odot Q_{2}=-V_{s}\left(Q_{2}\right) \odot Q_{1} .
$$

The skew-symmetric bracket operation

$$
[\cdot, \cdot]_{V_{s}}: \operatorname{Char}\left(T_{s}\right) \times \operatorname{Char}\left(T_{s}\right) \rightarrow \operatorname{Char}\left(T_{s}\right)
$$

enjoys the Jacobi identity making Charq $\left(T_{s}\right)$ into a Lie algebra. This is a particular case of the Lie bracket on characteristics introduced in $[14]^{3}$. Equation (4.4) says the map (4.3) defines a homomorphism from the Lie algebra of characteristics to the Lie algebra of characteristic symmetries. For low spins $(s=1 / 2,1)$ the Lie algebra $\operatorname{Char}\left(T_{s}\right)$ contains a finite dimensional subalgebra which is isomorphic to the Lie algebra of conformal group. The elements of this subalgebra correspond to conserved currents that are expressible in terms of the energy-momentum tensor.

Remark 2. The map (4.3) extends naturally from the space of characteristics to the space of all adjoint symmetries, keeping the same value area. Actually, this is a general property of any system of PDEs, and not just a special feature of the Bargmann-Wigner equations (see relation (A.6) in Appendix A). If one includes the adjoint symmetries of (2.1) to the range of definition, then the image of $V_{s}$ will cover all the symmetries of the system, except for a finite number of symmetries of order $\leq 2 s-1$. Although (A.6) has the form of a linear mapping, it should be better thought of as a universal bilinear map assigning a symmetry $Z$ to any pair $(V, P)$ constituted by a Lagrange anchor $V$ and an adjoint symmetry $P$. The map is universal as it does not depend on a particular structure of the field equations (A.1). In this context it is pertinent to mention another universal bilinear map connecting symmetries, adjoint symmetries, and characteristics [1,6]. This takes a symmetry $Z$ and an adjoint symmetry $P$

\footnotetext{
${ }^{3}$ For Lagrangian theories endowed with the canonical Lagrange anchor [15] this bracket reproduces the wellknown Dickey's bracket on conserved currents [8] (see also [5]).
} 
to a characteristic $Q$. For each specific choice of $P$ the assignment $(Z, P) \mapsto Q$ defines a linear mapping from the space of symmetries to the space of characteristics. This map, however, acts in the opposite direction with respect to the Lagrange anchor map (A.6). Either of the universal mappings can be regarded as an extension of the classical Noether's theorem to nonvariational PDEs, though the constructions are not entirely peer entities, in the following sense. For Lagrangian equations, there is a preferable choice for the Lagrange anchor that immediately reproduces the standard Noether's correspondence between symmetries and conservation laws. It is unlikely that such a canonical choice can exist for the adjoint symmetry of Lagrangian equations.

\section{$5 \quad$ Probability amplitude for massless fields of spin $s \geq 1 / 2$}

In this section, we briefly comment on how the Lagrange anchor above can be used to perform a consistent path-integral quantization of the massless fields subject to the Bargmann-Wigner equations.

Recall that in the covariant formulation of quantum field theory one usually deals with the path integrals of the form

$$
\langle\mathcal{O}\rangle_{\Psi}=\int[D \phi] \mathcal{O}[\phi] \Psi[\phi]
$$

where $\mathcal{O}$ is a physical observable and $\Psi$ is a probability amplitude on the configuration space of fields $\phi^{i}$. For a Lagrangian theory with action $S[\phi]$ the latter is given by the Feynman amplitude $\Psi=e^{\frac{i}{\hbar} S}$, which can also be defined as a unique (up to a normalization factor) solution to the Schwinger-Dyson (SD) equation

$$
\left(\frac{\delta S}{\delta \phi^{i}}+i \hbar \frac{\delta}{\delta \phi^{i}}\right) \Psi[\phi]=0
$$

After normalization, the integral (5.1) defines the quantum average of a physical observable $\mathcal{O}$ in the theory with probability amplitude $\Psi$. It is believed that evaluating such integrals for various observables $\mathcal{O}$ one can extract all physically relevant information about the quantum dynamics of fields (e.g. the scattering matrix).

In [22], the SD equation (5.2) was shown to admit quite a natural generalization to nonLagrangian theories endowed with compatible Lagrange anchors. Leaving aside general definitions, we just present the generalized SD equations for the field equations (2.1) and the Lagrange anchor (4.2). These read

$$
\begin{aligned}
& \left(T_{\alpha_{1} \ldots \alpha_{2 s-1}}^{\dot{\alpha}}-i^{2 s} \hbar \partial_{\alpha_{1} \dot{\alpha}_{1}} \cdots \partial_{\alpha_{2 s-1} \dot{\alpha}_{2 s-1}} \frac{\delta}{\delta \bar{\varphi}_{\dot{\alpha} \dot{\alpha}_{1} \ldots \dot{\alpha}_{2 s-1}}}\right) \Psi[\varphi]=0, \\
& \left(\bar{T}_{\dot{\alpha}_{1} \ldots \dot{\alpha}_{2 s-1}}^{\alpha}-i^{-2 s} \hbar \partial_{\alpha_{1} \dot{\alpha}_{1}} \cdots \partial_{\alpha_{2 s-1} \dot{\alpha}_{2 s-1}} \frac{\delta}{\delta \varphi_{\alpha \alpha_{1} \ldots \alpha_{2 s-1}}}\right) \Psi[\varphi]=0 .
\end{aligned}
$$

As with the usual SD equation (5.2), the first terms in (5.3) are determined by the classical equations of motion $T_{s}$ and the second ones, constructed by the Lagrange anchor $V_{s}$, involve the first-order variational derivatives multiplied by the Plank constant. In the classical limit, $\hbar \rightarrow 0$, the latter terms vanish and the resulting probability amplitude is given by the Dirac distribution supported at the classical solutions to the field equations. Formally, $\left.\Psi\right|_{\hbar \rightarrow 0} \sim \delta\left(T_{s}\right)$ and one can think of the last expression as a classical probability amplitude. The generalized SD equations (5.3) are formally consistent since the linear operators determining the left hand sides 
commute to each other ${ }^{4}$. The general solution to (5.3) admits a nice path-integral representation in terms of the action functional for the so-called augmented theory. Within the augmentation approach [21] the original set of fields $\varphi_{\alpha_{1} \ldots \alpha_{2 s}}$ is extended by the new fields $Y_{\dot{\alpha}}^{\alpha_{1} \ldots \alpha_{2 s-1}}$ that take values in the space dual to the space of equations of motion. The action of the augmented theory is systematically constructed by the original equations of motion and the corresponding Lagrange anchor. In the case at hand we find

$$
\begin{aligned}
S_{\text {aug }}[\varphi, Y]=\int d^{4} x & \left(Y_{\dot{\alpha}}^{\alpha_{1} \ldots \alpha_{2 s-1}} T_{\alpha_{1} \ldots \alpha_{2 s-1}}^{\dot{\alpha}}\right. \\
& \left.+i^{2 s} 2 \partial_{\alpha_{1}\left(\dot{\alpha}_{1}\right.} \cdots \partial_{\alpha_{2 s-1} \dot{\alpha}_{2 s-1}} Y_{\left.\dot{\alpha}_{2 s}\right)}^{\alpha_{1} \ldots \alpha_{2 s-1}} \partial^{\alpha_{2 s} \dot{\alpha}_{1}} \bar{Y}_{\alpha_{2 s}}^{\dot{\alpha}_{2} \ldots \dot{\alpha}_{2 s}}+\text { c.c. }\right) .
\end{aligned}
$$

The least action principle results in the system of decoupled equations

$$
\partial^{\alpha \dot{\alpha}} \varphi_{\alpha \alpha_{1} \ldots \alpha_{2 s-1}}=0, \quad \partial^{\left(\alpha_{1} \dot{\alpha}\right.} Y_{\dot{\alpha}}^{\left.\alpha_{2} \ldots \alpha_{2 s}\right)}=0 .
$$

The dynamics of $\varphi$ 's are seen to be governed by the original equations of motion (2.1). The equations for the augmentation fields $Y$ are known as the adjoint equations. The latter are the starting point for the construction of conserved currents by method [3].

Integrating by parts under the path-integral sign, one can easily verify that the functional

$$
\Psi[\varphi]=N \int[D Y] e^{\frac{i}{\hbar} S_{\operatorname{aug}}[\varphi, Y]}
$$

does satisfy (5.3) for an arbitrary normalization constant $N$. Doing the Gaussian integral (5.4), one can then find an explicit expression for the functional $\Psi$. As an example, consider the case $s=1 / 2$. The integral (5.4) takes the form

$$
\Psi[\varphi]=N \int[D Y] \exp \left(\frac{i}{\hbar} \int d^{4} x Y_{\dot{\alpha}} T^{\dot{\alpha}}-\bar{Y}_{\alpha} \bar{T}^{\alpha}+i Y_{\dot{\alpha}} \partial^{\alpha \dot{\alpha}} \bar{Y}_{\alpha}\right) .
$$

Making the substitution $Y_{\dot{\alpha}} \mapsto Y_{\dot{\alpha}}-i \bar{\varphi}_{\dot{\alpha}}$ and integrating by $Y$ 's, we obtain

$$
\Psi[\varphi]=N^{\prime} e^{\frac{i}{\hbar} S_{1 / 2}[\varphi]},
$$

where $N^{\prime}$ is some constant and

$$
S_{1 / 2}[\varphi]=-i \int d^{4} x \bar{\varphi}^{\dot{\alpha}} \partial_{\alpha \dot{\alpha}} \varphi^{\alpha}
$$

is the usual action for spin- $1 / 2$ massless field. Similar computations ${ }^{5}$ for $s>1 / 2$ result in the probability amplitude $\Psi \sim e^{\frac{i}{\hbar} S}$, where the exponent $S[\varphi]$ is no longer a local functional. An explicit expression for the spin-1 non-local action $S_{1}[\varphi]$ can be found in [21].

Notice that the action of the augmented theory is manifestly Poincaré invariant for any spin. This suggests that the relativistic symmetries presumably survive quantization. It would be interesting to establish which higher symmetries of the original field equations can be "lifted" to the augmented theory and then to quantum theory. Another interesting problem is to classify all nontrivial Lagrange anchors for the Bargmann-Wigner equations (2.1). This problem is in principle of the same kind as the classification of symmetries or characteristics, though no explicit examples are known except for the Lagrange anchor considered in the present paper. This seems to be a unique Lagrange anchor enjoying the Poincaré invariance.

\footnotetext{
${ }^{4}$ In fact, it is the requirement of formal compatibility of the generalized SD equations that determines all possible Lagrange anchors for given equations of motion.

${ }^{5}$ Notice that the Noether identities (2.2) give rise to certain gauge symmetries in the augmented theory [21], namely, $\delta_{\varepsilon} Y_{\dot{\alpha}}^{\alpha_{1} \ldots \alpha_{2 s-1}}=\partial_{\dot{\alpha}}^{\left(\alpha_{1}\right.} \varepsilon^{\left.\alpha_{2} \ldots \alpha_{2 s-1}\right)}, \delta_{\varepsilon} \varphi=0$. Therefore, to compute the Gaussian integral (5.4) for $s>1 / 2$ one should first impose an appropriate gauge fixing condition on $Y$ 's.
} 


\section{Conclusion}

In this paper, we have found a Poincaré invariant Lagrange anchor for the Bargmann-Wigner equations and applied this anchor for deriving symmetries from conservation laws and for defining the quantum probability amplitude. Of course, at the level of free fields there is an equivalent Lagrangian formulation due to Fang and Fronsdal [11, 12]. This formulation allows one to solve the above problems by standard tools of Lagrangian field theory. The Lagrangian formulation, however, does not admit consistent interactions, while the non-Lagrangian equations for interacting higher-spin fields have been proposed by Vasiliev [30, 31]. The Lagrange anchor for Vasiliev's equations is still unknown. If the anchor is found, it will allow one to develop a consistent quantum theory of higher-spin interactions. There are many other non-Lagrangian models of physical interest for which the concept of Lagrange anchor seems having no alternatives when it comes to quantization or establishing a systematic connection between symmetries and conservation laws. The procedure of finding the Lagrange anchor in the considered simple model is quite general and it can be instructive in more complex theories.

\section{A General definition and properties of a Lagrange anchor}

To make the paper self-contained, we explain here some basic notions concerning the concept of Lagrange anchor. A more extended and systematic exposition can be found in the original papers $[14,15,21]$. To emphasize the algebraic structure underlying the concept, we use De Witt's condensed notation [7], which is much more handy and compact than the jet space formalism, given the context. According to this notation the fields $\phi^{i}(x)$, leaving on a smooth manifold $X$, are interpreted as local coordinates on an infinite dimensional supermanifold $M$. The local coordinates $x$ 's on $X$ are treated as continuous indices labeling the fields and included into a singe superindex " $i$ ", so that $\phi^{i} \equiv \phi^{i}(x)$. The repeated superindex implies summation over its discrete part and integration over $X$ with respect to an appropriate measure. The partial derivatives with respect to $\phi^{i}$ are understood as functional ones, that is, $\partial_{i}=\delta / \delta \phi^{i}(x)$. The role of the space of smooth functions $C^{\infty}(M)$ is played by the space of local functionals of fields; in so doing, two functionals are considered to be equivalent if they differ by boundary terms. In particular, the equality $S(\phi)=0$ implies that the local functional $S$ is given by the integral of a total divergence. Notice that unlike the smooth functions on a finite dimensional supermanifold, the local functionals do not form a supercommutative algebra.

With the condensed notation, any system of field equations can be written as

$$
T_{a}(\phi)=0 .
$$

As we do not assume the field equations to come from the least action principle, the discrete parts of the superindices $i$ and $a$, labelling respectively the components of fields and equations, may run through completely different sets. For example, the relativistic wave equations (2.1) take their values in the spin-tensor fields of type $(s-1,1)$, while the corresponding fields constitute a spin-tensor of type $(s, 0)$. In this case, the infinite dimensional supermanifold $M$ of spintensor fields is purely bosonic (i.e., just a manifold) for integer spin and purely fermionic for half-integer ones. Continuing the geometric interpretation above, one can regard the differential operators $T$ 's as components of a section $T=\left\{T_{a}\right\}$ of some infinite dimensional supervector bundle $\mathcal{E}$ over the base supermanifold $M$. In [15], it was proposed to call $\mathcal{E} \rightarrow M$ the dynamics bundle. Then the solutions to the field equations (A.1) are identified with the zero locus $\Sigma \subset M$ of the section $T$, the shell in the physical terminology. The field equations (A.1) are called regular if any local function of fields that vanishes on $\Sigma$ is proportional to the local functions $T_{a}$

and their derivatives with respect to the local coordinates on $X$. Below we assume that the equations (A.1) are regular. The functional derivative of $T$ 's gives the operator $J_{i a}=\partial_{i} T_{a}$ 
defining the so-called universal linearization of the equations (A.1). It can also be viewed as an operator defining a homomorphism $J: T M \rightarrow \mathcal{E}$ from the tangent bundle of the space of fields $M$ to the dynamics bundle, if one regards $\partial_{i}$ as a covariant derivative associated to a flat connection in $\mathcal{E}$.

A vector field $Z=Z^{i} \partial_{i}$ on $M$, i.e., a section of the tangent bundle $T M$, is called a symmetry of the field equations (A.1) if

$$
\left.J(Z)\right|_{\Sigma}=0 \quad \Leftrightarrow \quad Z^{i} J_{i a}=A_{a}^{b} T_{b}
$$

for some $A$ 'a. The symmetries form a subalgebra in the Lie superalgebra of all vector fields on $M$. Let $\mathcal{E}^{*}$ denote the vector bundle dual to the dynamics bundle $\mathcal{E}$. A section $P=\left\{P^{a}\right\}$ of $\mathcal{E}^{*}$ is called an adjoint symmetry if

$$
\left.J^{*}(P)\right|_{\Sigma}=0 \quad \Leftrightarrow \quad J_{i a} P^{a}=B_{i}^{b} T_{b}
$$

for some $B$ 's. Among the adjoint symmetries one can extract those originating from the identities. A section $Q$ of $\mathcal{E}^{*}$ is said to generate an identity for the equations (A.1) if

$$
T_{a} Q^{a}=0 .
$$

As we have explained above, the last equality should be understood in the sense that the local functional of fields $T_{a} Q^{a}$ is given by the integral of a total divergence div $j$. By definition, the current $j$ is conserved when evaluated on solutions to (A.1), that is, $\left.\operatorname{div} j\right|_{\Sigma}=0$. Neither identities nor conserved currents are defined by relation (A.3) uniquely. The equivalence classes of identities that correspond to equivalence classes of nontrivial conserved currents are called characteristics. This gives a linear bijection between the spaces of characteristics and nontrivial conserved currents. Taking the functional derivative of both the sides of equality (A.3), one can see that each identity $Q$ satisfies the adjoint symmetry condition (A.2).

Consider now a homomorphism $V: \mathcal{E}^{*} \rightarrow T M$, where $V$ is assumed to be defined, like the universal linearization $J$, by some matrix differential operator whose coefficients are local functions of fields. By definition, the homomorphism $V$ is called a Lagrange anchor, if the following diagram of maps becomes supercommutative upon restriction to $\Sigma$ :

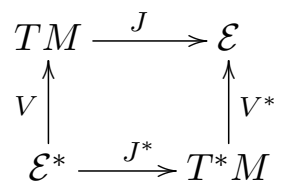

For the regular equations (A.1), the last condition can be written explicitly as

$$
V_{a}^{i} \partial_{i} T_{b}-(-1)^{\epsilon_{a} \epsilon_{b}} V_{b}^{i} \partial_{i} T_{a}=C_{a b}^{d} T_{d}
$$

for some $C$ 's. Here $\epsilon_{a} \in \mathbb{Z}_{2}$ denotes the Grassmann parity of the local function of fields $T_{a}$. In the particular case of Lagrangian equations, $T_{i}=\partial_{i} S=0$, the dynamics bundle coincides with the cotangent bundle $T^{*} M$ and (A.5) is satisfied with the identity map $V=\mathrm{id}: T M \rightarrow T M$ and all $C$ 's vanishing. This is known as the canonical Lagrange anchor for Lagrangian equations. Notice that even in the Lagrangian situation the system (A.5) may admit a lot of other (noncanonical) solutions. Like symmetries and adjoint symmetries, all they form a vector superspace.

By definition, the spaces of symmetries and adjoint symmetries are identified with the onshell kernels of the horizontal maps in (A.4). Form the on-shell supercommutativity of the diagram it then follows that the vector field $Z=V(P)$ is a symmetry of (A.1) for any adjoint symmetry $P$. Thus, each Lagrange anchor $V$ gives rise to a homomorphism

$$
V: \operatorname{AdSym}(T) \rightarrow \operatorname{Sym}(T)
$$

acting from the space of adjoint symmetries to the space of symmetries of the equations (A.1). 
A Lagrange anchor $V$ is said to be strongly integrable ${ }^{6}$ if the following two conditions are satisfied

$$
\left[V_{a}, V_{b}\right]=C_{a b}^{d} V_{d}, \quad(-1)^{\epsilon_{a} \epsilon_{c}}\left(C_{a b}^{d} C_{c d}^{e}+V_{c}^{i} \partial_{i} C_{a b}^{e}\right)+\operatorname{cycle}(a, b, c)=0 .
$$

The first equation means that $\operatorname{Im} V \subset T M$ is an integrable distribution on $M$. In case Ker $V=0$, the second condition in (A.7) follows from the first one by the Jacobi identity for the supercommutator of the local vector fields $V_{a}=V_{a}^{i} \partial_{i}$. The integrability conditions (A.7) have a nice geometric interpretation as defining a Lie superalgebroid over $M$. For a general discussions of Lie algebroids we refer the reader to [23]. Upon this interpretation the Lagrange anchor is identified with the anchor of a Lie superalgebroid $V: \mathcal{E}^{*} \rightarrow T M$ and the Lie superalgebra structure on the sections of $\mathcal{E}^{*}$ is defined by the bracket

$$
\left[e_{a}, e_{b}\right]=C_{a b}^{d} e_{d}
$$

where $\left\{e_{a}\right\}$ are frame sections in $\mathcal{E}^{*}$. The defining relation (A.5) for the Lagrange anchor can then be reinterpreted as the closedness condition for the $1-\mathcal{E}$-form $T$ with respect to the Lie algebroid differential, $d_{\mathcal{E}} T=0$; in so doing, the integrability condition (A.7) is expressed by the operator equality $d_{\mathcal{E}}^{2}=0$. It is not hard to see [14] that for a strongly integrable Lagrange anchor the generators of identities (A.3) form a subalgebra in the full Lie algebra of sections of $\mathcal{E}^{*}$. The canonical Lagrange anchor of Lagrangian equations gives an example of strongly integrable Lagrange anchor. It corresponds to the tangent Lie algebroid id :TM $\rightarrow T M$. Although the theory of Lie algebroids and groupoids is a fascinating area of modern differential geometry, it by no means covers or substitutes the concept of a Lagrange anchor completely, as the strong integrability condition (A.7) is too restrictive and is not actually needed in many field-theoretical applications.

\section{B Lagrange anchor for the Bargmann-Wigner equations}

Unfolding the condensed notation of Appendix A, one can see that the universal linearization of the field equations (2.1) and the Lagrange anchor (4.2) are defined by the following operators:

$$
\begin{aligned}
& J_{\alpha_{1} \ldots \alpha_{2 s-1}}^{\beta \beta_{1} \ldots \beta_{2 s-1}, \dot{\alpha}}(x, z)=\delta_{\alpha_{1}}^{\left(\beta_{1}\right.} \cdots \delta_{\alpha_{2 s-1}}^{\beta_{2 s-1}}\left(\partial^{z}\right)^{\dot{\alpha} \beta)} \delta(x-z), \\
& V_{\dot{\gamma}_{1} \ldots \dot{\gamma}_{2 s-1}, \beta \beta_{1} \ldots \beta_{2 s-1}}^{\gamma}(z, y)=(-i)^{2 s} \delta_{(\beta}^{\gamma}\left(\partial^{z}\right)_{\beta_{1} \dot{\gamma}_{1}} \cdots\left(\partial^{z}\right)_{\left.\beta_{2 s-1}\right) \gamma_{2 s-1}} \delta(z-y) .
\end{aligned}
$$

There are also operators corresponding to the complex conjugate equations (2.1). Their integral kernels are obtained by complex conjugation of (B.1).

Since both the operators in (B.1) are independent of fields, the defining property for the Lagrange anchor is fulfilled iff the left hand side of (A.5) is equal to zero identically, that is, all $C$ 's must vanish. This amounts to the equality

$$
\int d^{4} z J^{\beta \beta_{1} \ldots \beta_{2 s-1}, \dot{\alpha}}{ }_{\alpha_{1} \ldots \alpha_{2 s-1}}(x, z) V_{\dot{\gamma}_{1} \ldots \dot{\gamma}_{2 s-1}, \beta \beta_{1} \ldots \beta_{2 s-1}}(z, y)-(i)^{4 s} \overline{(\alpha \leftrightarrow \gamma, x \leftrightarrow y)}=0 .
$$

On substituting (B.1) into (B.2), we get

$$
\left\{\left(\partial^{y}\right)^{\gamma \dot{\alpha}}\left(\partial^{y}\right)_{\alpha_{1}\left(\dot{\gamma}_{1}\right.} \cdots\left(\partial^{y}\right)_{\left.\alpha_{2 s-1} \dot{\gamma}_{2 s-1}\right)} \delta(x-y)-(-1)^{2 s}(x \leftrightarrow y)\right\}
$$

\footnotetext{
${ }^{6}$ In [14], we used the term integrable instead of strongly integrable. A more relaxed notion of integrability was formulated in our recent paper [16]. To distinguish between these two versions of integrability, from now on we reserve the term integrable for the Lagrange anchors satisfying the relaxed integrability condition in the sense of $[16]$.
} 


$$
\begin{aligned}
& +\left[\sum_{p=1}^{2 s-1} \delta_{\alpha_{p}}^{\gamma}\left(\partial^{y}\right)^{\beta \dot{\alpha}}\left(\partial^{y}\right)_{\beta \dot{\gamma}_{p}}\left(\partial^{y}\right)_{\alpha_{1}\left(\dot{\gamma}_{1}\right.} \cdots\left(\partial^{y}\right)_{\alpha_{p-1} \dot{\gamma}_{p-1}}\left(\partial^{y}\right)_{\alpha_{p+1} \dot{\gamma}_{p+1}} \cdots\left(\partial^{y}\right)_{\left.\alpha_{2 s-1} \dot{\gamma}_{2 s-1}\right)} \delta(x-y)\right. \\
& \left.-(-1)^{2 s}(\alpha \leftrightarrow \dot{\gamma}, \dot{\alpha} \leftrightarrow \gamma, x \leftrightarrow y)\right]=0
\end{aligned}
$$

Due to the symmetry properties of the derivatives of Dirac's $\delta$-function, the terms in the braces cancel each other. Transferring now all the partial derivatives in the square brackets to $y$ 's and using the identities

$$
\left(\partial^{y}\right)^{\beta \dot{\alpha}}\left(\partial^{y}\right)_{\beta \dot{\gamma}_{p}}=\frac{1}{2} \delta_{\dot{\gamma}_{p}}^{\dot{\alpha}}\left(\partial^{y}\right)^{\beta \dot{\beta}}\left(\partial^{y}\right)_{\beta \dot{\beta}}, \quad\left(\partial^{y}\right)^{\gamma \dot{\beta}}\left(\partial^{y}\right)_{\alpha_{p} \dot{\beta}}=\frac{1}{2} \delta_{\alpha_{p}}^{\gamma}\left(\partial^{y}\right)^{\beta \dot{\beta}}\left(\partial^{y}\right)_{\beta \dot{\beta}},
$$

one can bring the left hand side of (B.3) to the form

$$
\begin{aligned}
& \frac{1}{2}\left\{\sum_{p=1}^{2 s-1}\left(\delta_{\alpha_{p}}^{\gamma} \delta_{\left(\dot{\gamma}_{p}\right.}^{\dot{\alpha}}-\delta_{\alpha_{p}}^{\gamma} \delta_{\left(\dot{\gamma}_{p}\right.}^{\dot{\alpha}}\right)\left(\partial^{y}\right)^{\beta \dot{\beta}}\left(\partial^{y}\right)_{\beta \dot{\beta}}\right. \\
& \left.\quad \times\left(\partial^{y}\right)_{\alpha_{1} \dot{\gamma}_{1}} \cdots\left(\partial^{y}\right)_{\alpha_{p-1} \dot{\gamma}_{p-1}}\left(\partial^{y}\right)_{\alpha_{p+1} \dot{\gamma}_{p+1}} \cdots\left(\partial^{y}\right)_{\left.\alpha_{2 s-1} \dot{\gamma}_{2 s-1}\right)} \delta(x-y)\right\} .
\end{aligned}
$$

The last expression is equal to zero identically, and verification of the defining property (A.5) for the Lagrange anchor (4.2) is completed.

\section{Acknowledgments}

We are thankful to G. Barnich, and E. Skvortsov for illuminating discussions on various issues related to the subject of this paper and for relevant references. We also benefited from the valuable comments by S. Anco and three anonymous referees that helped us to improve the initial version of the manuscript. The work was initiated during our visit to the Erwin Shrödinger Institute (Vienna, October-November 2010) and we appreciate its hospitality. The work was partially supported by the RFBR grant No 09-02-00723-a and also by Russian Federal Agency of Education under the State Contract no P789. AAS appreciates the financial support from Dynasty Foundation, SLL acknowledges partial support from the RFBR grant 11-01-00830-a.

\section{References}

[1] Anco S.C., Bluman G., Direct construction of conservation laws from field equations, Phys. Rev. Lett. 78 (1997), 2869-2873.

[2] Anco S.C., Pohjanpelto J., Classification of local conservation laws of Maxwell's equations, Acta Appl. Math. 69 (2001), 285-327, math-ph/0108017.

[3] Anco S.C., Pohjanpelto J., Conserved currents of massless fields of spin $s \geq \frac{1}{2}$, R. Soc. Lond. Proc. Ser. A Math. Phys. Eng. Sci. 459 (2003), 1215-1239, math-ph/0202019.

[4] Barnich G., Brandt F., Henneaux M., Local BRST cohomology in the antifield formalism. I. General theorems, Comm. Math. Phys. 174 (1995), 57-91, hep-th/9405109.

[5] Barnich G., Henneaux M., Isomorphisms between the Batalin-Vilkovisky antibracket and the Poisson bracket, J. Math. Phys. 37 (1996), 5273-5296, hep-th/9601124.

[6] Bluman G.W., Cheviakov A.F., Anco S.C., Applications of symmetry methods to partial differential equations, Applied Mathematical Sciences, Vol. 168, Springer, New York, 2010.

[7] DeWitt B.S., Dynamical theory of groups and fields, Gordon and Breach Science Publishers, New York, 1965.

[8] Dickey L.A., Soliton equations and Hamiltonian systems, Advanced Series in Mathematical Physics, Vol. 12, World Scientific Publishing Co. Inc., River Edge, NJ, 1991. 
[9] Dubois-Violette M., Henneaux M., Talon M., Viallet C.M., Some results on local cohomologies in field theory, Phys. Lett. B $\mathbf{2 6 7}$ (1991), 81-87.

[10] Fairlie D.B., Conservation laws and invariance principles, Nuovo Cimento 37 (1965), 897-904.

[11] Fang J., Fronsdal C., Massless fields with half-integral spin, Phys. Rev. D 18 (1978), 3630-3633.

[12] Fronsdal C., Massless fields with integer spin, Phys. Rev. D 18 (1978), 3624-3629.

[13] Fushchich W.I., Nikitin A.G., Symmetries of equations of quantum mechanics, Allerton Press Inc., New York, 1994.

[14] Kaparulin D.S., Lyakhovich S.L., Sharapov A.A., Rigid symmetries and conservation laws in non-Lagrangian field theory, J. Math. Phys. 51 (2010), 082902, 22 pages, arXiv:1001.0091.

[15] Kazinski P.O., Lyakhovich S.L., Sharapov A.A., Lagrange structure and quantization, J. High Energy Phys. 2005 (2005), 076, 42 pages, hep-th/0506093.

[16] Kazinski P.O., Lyakhovich S.L., Sharapov A.A., Local BRST cohomology in (non-)Lagrangian field theory, J. High Energy Phys. 2011 (2011), 006, 34 pages, arXiv:1106.4252.

[17] Kibble T.W.B., Conservation laws for free fields, J. Math. Phys. 6 (1965), 1022-1026.

[18] Konstein S.E., Vasiliev M.A., Zaikin V.N., Conformal higher spin currents in any dimension and AdS/CFT correspondence, J. High Energy Phys. 2000 (2000), 018, 12 pages, hep-th/0010239.

[19] Kosmann-Schwarzbach Y., The Noether theorems, Sources and Studies in the History of Mathematics and Physical Sciences, Springer, New York, 2011.

[20] Lipkin D.M., Existence of a new conservation law in electromagnetic theory, J. Math. Phys. 5 (1964), 696-700.

[21] Lyakhovich S.L., Sharapov A.A., Quantizing non-Lagrangian gauge theories: an augmentation method, J. High Energy Phys. 2007 (2007), 047, 40 pages, hep-th/0612086.

[22] Lyakhovich S.L., Sharapov A.A., Schwinger-Dyson equation for non-Lagrangian field theory, J. High Energy Phys. 2006 (2006), 007, 27 pages, hep-th/0512119.

[23] Mackenzie K.C.H., General theory of Lie groupoids and Lie algebroids, London Mathematical Society Lecture Note Series, Vol. 213, Cambridge University Press, Cambridge, 2005.

[24] Morgan T.A., Two classes of new conservation laws for the electromagnetic field and for other massless fields, J. Math. Phys. 5 (1964), 1659-1660.

[25] Năstăsescu C., Van Oystaeyen F., Graded and filtered rings and modules, Lecture Notes in Mathematics, Vol. 758, Springer, Berlin, 1979.

[26] Olver P.J., Applications of Lie groups to differential equations, Graduate Texts in Mathematics, Vol. 107, Springer-Verlag, New York, 1986.

[27] Penrose R., Rindler W., Spinors and space-time, Vols. I and II, Cambridge University Press, Cambridge, 1987.

[28] Pohjanpelto J., Anco S.C., Generalized symmetries of massless free fields on Minkowski space, SIGMA 4 (2008), 004, 17 pages, arXiv:0801.1892.

[29] Streater R.F., Wightman A.S., PCT, spin and statistics, and all that, W.A. Benjamin, Inc., New York Amsterdam, 1964.

[30] Vasiliev M.A., Consistent equations for interacting gauge fields of all spins in $3+1$ dimensions, Phys. Lett. B 243 (1990), 378-382.

[31] Vasiliev M.A., Higher spin gauge theories in various dimensions, Fortschr. Phys. 52 (2004), 702-717, hep-th/0401177.

[32] Vasiliev M.A., Gelfond O.A., Skvortsov E.D., Higher-spin conformal currents in Minkowski space, Theoret. Math. Phys. 154 (2008), 294-302, hep-th/0601106. 